\title{
Role of cross-talk between the Smad2 and MAPK pathways in TGF-B1-induced collagen IV expression in mesangial cells
}

\author{
WEINA JIANG ${ }^{1}$, YAN ZHANG ${ }^{1}$, HUIJUAN WU ${ }^{1,2}$, XIN ZHANG $^{1}$, HUALEI GAN ${ }^{1}$, \\ JIANYONG SUN $^{1}$, QI CHEN ${ }^{1}$, MUYI GUO ${ }^{1}$ and ZHIGANG ZHANG ${ }^{1,2}$ \\ ${ }^{1}$ Department of Pathology, ${ }^{2}$ Key Laboratory of Molecular Medicine, Ministry of Education of China, \\ Shanghai Medical College, Fudan University, Shanghai 200032, P.R. China
}

Received April 20, 2010; Accepted June 18, 2010

DOI: 10.3892/ijmm_00000501

\begin{abstract}
Transforming growth factor $\beta 1$ (TGF-ß1) can promote sclerosis in many kidney diseases by enhancing the synthesis of collagens. However, the mechanisms of downstream intracellular signal transduction in TGF-ß1-induced collagen synthesis is not fully understood. The purpose of this study was to further investigate the mechanisms and the cross-talk between the MAPK and Smad2 pathways. We found that U0126, a specific inhibitor of ERK1/2, and SB203580, a specific inhibitor of p38, down-regulated the TGF-ß1-induced phosphorylation of Smad2 at both linker and C-terminal sites in rat mesangial cells. Whereas, SP600125, a specific inhibitor of JNK, only down-regulated the phosphorylation of Smad2 at the C-terminal sites, but had little effect on the phosphorylation of Smad2 at linker sites. However, all three MAPK inhibitors reduced collagen IV synthesis induced by TGF- $\$ 1$. Furthermore, TGF- $\$ 1$ induced the phosphorylation of Smad2 at both the linker and C-terminal sites. Transient transfection of a dominant negative Smad2 construct significantly decreased TGF-ß1-induced phosphorylation of ERK1/2, JNK and expression of collagen IV, but did not decrease the phosphorylation of $\mathrm{p} 38$. These findings demonstrate that there is cross-talk between the MAPK (ERK1/2, JNK, p38) and Smad2 pathways, and that the cross-talk interacts mutually to enhance the synthesis of collagen IV in rat mesangial cells.
\end{abstract}

\section{Introduction}

Glomerulosclerosis, which is the result of structural remodeling and excessive deposition of extracellular matrix (ECM) in the glomerulus, is very common in end-stage glomerulonephritis. Mesangial cells (MCs) have been identified as the main

Correspondence to: Professor Zhigang Zhang, Department of Pathology and Key Laboratory of Molecular Medicine, Ministry of Education of China, Shanghai Medical College, Fudan University, 138 Yixueyuan Road, Shanghai 200032, P.R. China

E-mail: zzg@shmu.edu.cn

Key words: transforming growth factor- $\beta 1$, Smad, mitogenactivated protein kinase, cross-talk, sclerosis producer of ECM and contribute critically to the structural and functional integrity of the glomerulus (1).

It is well known that transforming growth factor $\beta 1$ (TGF-B1) plays a crucial role in the sclerosis associated with progressive renal diseases (2). TGF- $\beta 1$ can cause ECM accumulation by enhancing the production of collagen and fibronectin in MCs (3), suppressing the expression of ECMdegrading proteases (4), or increasing the synthesis of ECM protease inhibitors (5). The Smad family of proteins are classic signal transducers downstream of TGF- 31 . Other signaling molecules, such as MAPK and AKT/PI3K, have also been reported to play important roles in signal transduction downstream of TGF- $\beta 1(6,7)$.

In the past decade, many studies have highlighted crosstalk among different signaling pathways as a powerful means of coordinating signaling processes, exerting synergistic or antagonistic effects on the regulation of the transcription activity of target genes (8-10). Increasing evidence has suggested a more complex paradigm of TGF- 11 -initiated signaling via Smads interacting with the MAPK pathway, which is a better elucidated pathway downstream of TGF- $\beta 1$ besides the classic Smad pathway. Li et al (11) reported that ERK2 enhanced the TGF-B1-induced synthesis of type I and III collagen in fibroblasts, accompanied by the activation of Smad2 linker sites. The ablation of ERK2 down-regulated significantly the synthesis of collagen type I and III (12). In cardiac fibroblasts, cAMP can inhibit TGF- $\beta 1$-induced collagen synthesis via inhibition of ERK1/2 and Smad signaling (13). The Matsuzaki laboratory demonstrated that activating JNK induced by TGF- $B 1$ can cause further phosphorylation of the linker and C-terminal sites of both Smad2 and Smad3, thereby disturbing the growth and invasion of rat gastric tumor cells $(14,15)$. Moris et al showed that JNK-dependent phosphorylation of Smad2 and Smad3 in response to HGF, PDGF and TGF- $\beta$, can transactivate target genes with no loss of R-Smad capacity (16). The suppression of JNK MAPK signaling by the deletion of Smad3 inhibits v-Ras-induced transformation in primary mouse embryonic fibroblasts (17). Furthermore, the p38 pathway is activated by TGF- $\beta 1$, and then stimulates Smaddependent transcription through SUMO-1-mediated modification of Smad4 in NIH3T3 and COS7 cells (18). In pancreatic cells, TGF- $\beta 1$-induced expression of biglycan required the activation of MKK6-p38 MAPK, that proved to 
be downstream of Smad signaling (19). The mechanism of these coordinating signaling processes, however, is not fully understood.

It has been found that both the Smads and MAPK are activated by TGF- $\beta 1$ in MCs during glomerulonephritis and the development of glomerulosclerosis (20). Only a few laboratories have considered the interaction of the two pathways in MCs. Hayashida et al reported that ERK-dependent phosphorylation of the Smad3 linker region increased collagen I synthesis in human MCs (21). The complex mechanisms of the interaction of these two signaling pathways still need to be further elucidated. In the present study, we investigated the cross-talk between the MAPK (ERK1/2, JNK, p38 MAPK) and Smad2 pathways, and determined the role of cross-talk in TGF-ß1-induced synthesis of collagen IV in rat MCs.

\section{Materials and methods}

Reagents. Recombinant human TGF- $\$ 1$ was purchased from R\&D Systems (Minneapolis, MN). Polyclonal antibodies against p38 and phospho-p38 MAPK, JNK and phosphoJNK, ERK1/2 and phospho-ERK1/2, and U0126 were obtained from Cell Signaling Technologies (Danvers, MA). SB203580 and SP600125 were purchased from Calbiochem (La Jolla, CA). Polyclonal antibody against type IV collagen was from ABcam (Cambridge, UK). Lipofectamine ${ }^{\mathrm{TM}} 2000$ was from Invitrogen (Carlsbad, CA).

Rat MC culture. Rat MCs were isolated and characterized as described previously (22). Cultures of rat MCs were established and maintained in DMEM (Gibco, Grand Island, NY) supplemented with $10 \%$ fetal bovine serum (Gibco), $100 \mathrm{U} / \mathrm{ml}$ penicillin, and $100 \mu \mathrm{g} / \mathrm{ml}$ streptomycin, $2 \mathrm{mM}$ glutamine and incubated in a $37^{\circ} \mathrm{C}$ humidified incubator with $5 \% \mathrm{CO}_{2}$. The cells used in this experiment were at passages 6-10. To evaluate the effect of TGF- 31 , cells were rendered quiescent in medium containing $0.5 \%$ fetal bovine serum for $20 \mathrm{~h}$, followed by treatment with $2.5 \mathrm{ng} / \mathrm{ml} \mathrm{TGF}-\beta 1$ for the indicated time periods. To evaluate the effects of MAPK inhibitors, cells were pretreated with inhibitors for $2 \mathrm{~h}$ after quiescence, and then treated with $2.5 \mathrm{ng} / \mathrm{ml}$ TGF- $\beta 1$ for the indicated time periods.

Transient transfection of rat MCs. The dominant negative Smad2 construct was a kind gift from Chun Peng (Department of Biology, York University, Canada). Transfection of rat MCs was carried out using Lipofectamine ${ }^{\mathrm{TM}} 2000$ reagent, according to the manufacturer's instructions. Control cells were transfected with an empty vector PcDNA3.1 alone. Cells were cultured for $48 \mathrm{~h}$ after transfection, and then treated with $2.5 \mathrm{ng} /$ $\mathrm{ml}$ TGF- 31 , with serum starvation of $20 \mathrm{~h}$ before treatment. Real-time quantitative RT-PCR and Western blot analyses were carried out after the treatment.

Western blot analysis. Cells were lysed in lysis buffer containing 1\% Nonidet P-40, $20 \mathrm{mM}$ Tris (pH 8.0), $150 \mathrm{mM}$ $\mathrm{NaCl}, 1 \mathrm{mM}$ phenylmethylsulfonyl fluoride (PMSF), $1 \mu \mathrm{g} / \mathrm{ml}$ aprotinin, $1 \mu \mathrm{g} / \mathrm{ml}$ leupeptin, $1 \mu \mathrm{g} / \mathrm{ml}$ pepstatin, $1 \mathrm{mM}$ $\mathrm{Na}_{3} \mathrm{VO}_{4}$, and $1 \mathrm{mM} \mathrm{NaF}$ on ice for $1 \mathrm{~h}$. Equal amounts of protein were separated by SDS-PAGE, using a $10 \%$ gel and
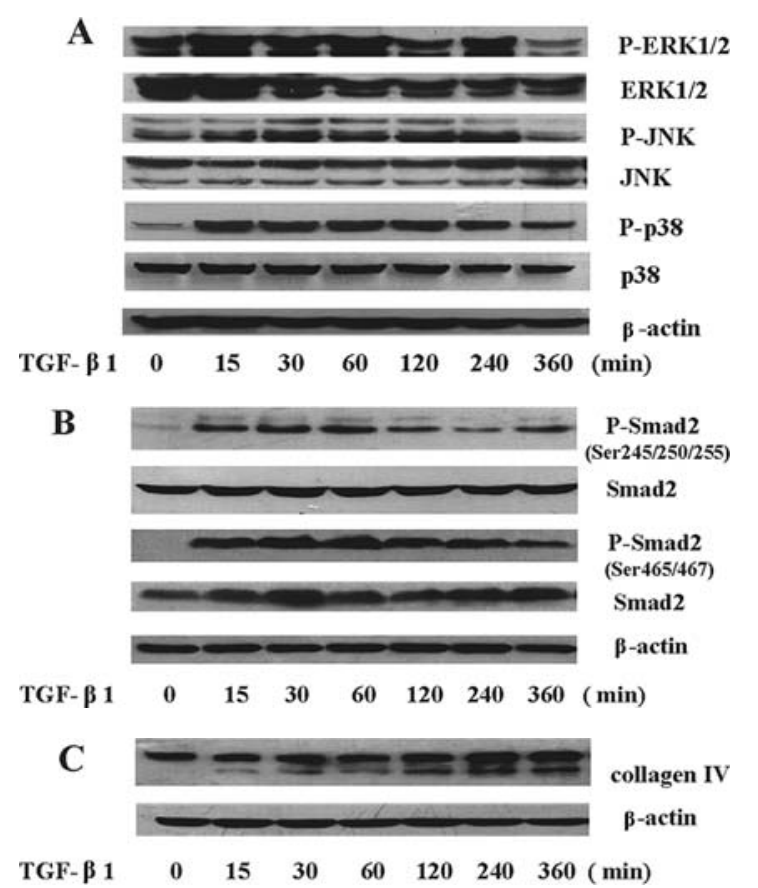

Figure 1. TGF- 31 induced the activation of ERK, JNK, p38, Smad2 and expression of collagen IV in MCs. Cells were stimulated with TGF- 11 $(2.5 \mathrm{ng} / \mathrm{ml})$ for the indicated times. (A) The relative ERK1/2 and phospho(P)-ERK1/2, JNK and P-JNK, p38 and P-p38 protein levels were determined by Western blotting. (B) The relative levels of Smad 2 and P-Smad2 phosphorylated at linker (Ser245/250/255) and C-terminal (Ser465/467) sites, and (C) collagen IV, determined by Western blotting. $\beta$-actin was used as a loading control. Results are representative of at least three independent experiments.

electro-transferred to polyvinylidene difluoride membranes (Millipore, Billerica, MA). After blocking with 5\% non-fat milk, membranes were incubated with appropriate primary antibodies overnight on a rocker at $4^{\circ} \mathrm{C}$. A monoclonal antiB-actin antibody (Sigma, St. Louis, MO) was used as control. After washing, membranes were incubated with HRPconjugated IgG (ProteinTech Group, Chicago, IL) for $1 \mathrm{~h}$, and target protein bands were visualized by chemiluminescence (Pierce, Rockford, IL).

Real-time quantitative RT-PCR. Total RNA was isolated from MCs using the Trizol reagent (Invitrogen, Carlsbad, CA). The isolated RNA was converted into cDNAs using the PrimeScript $^{\text {TM }}$ RT reagent Kit (Takara, Otsu, Shiga, Japan). Real-time quantitative RT-PCR was performed using a SYBR Premix Ex Taq ${ }^{\mathrm{TM}}$ Kit (Takara), in a total reaction volume of $20 \mu \mathrm{l}$ containing $10 \mu \mathrm{l}$ of SYBR Green RT-PCR master mix, $2 \mu \mathrm{l}$ of cDNA template and $0.4 \mu \mathrm{M}$ of each target-specific primer designed to amplify a part of each gene. After PCR, a melting curve analysis was performed to demonstrate the specificity of each PCR product as a single peak. The collagen IV gene expression levels were normalized by the corresponding gene expression levels of rat $\beta$-actin. Primer sets were as follows, rat collagen IV, 5'-TCCTTGTGACCA GGCATAGT-3' and 5'-TTGAACATCTCGCTCCTCTC-3'; rat $B$-actin, 5'-AGGATGCAGAAGGAGATTACTGC-3' and 5'-AAAACGCAGCTCAGTAACAGTGC-3'.

Statistical analysis. The statistical significance of the experimental data from three independent experiments was 
A

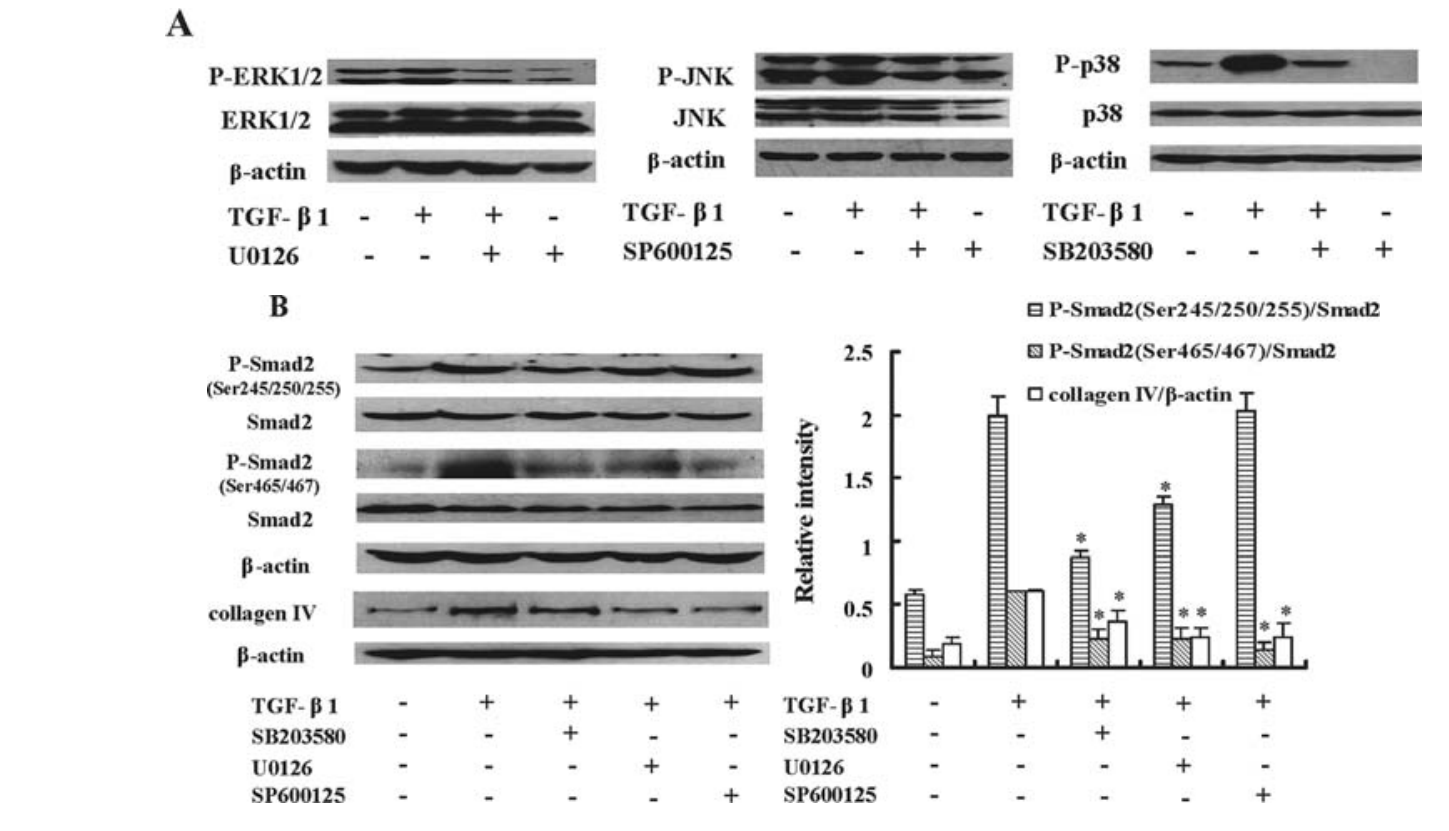

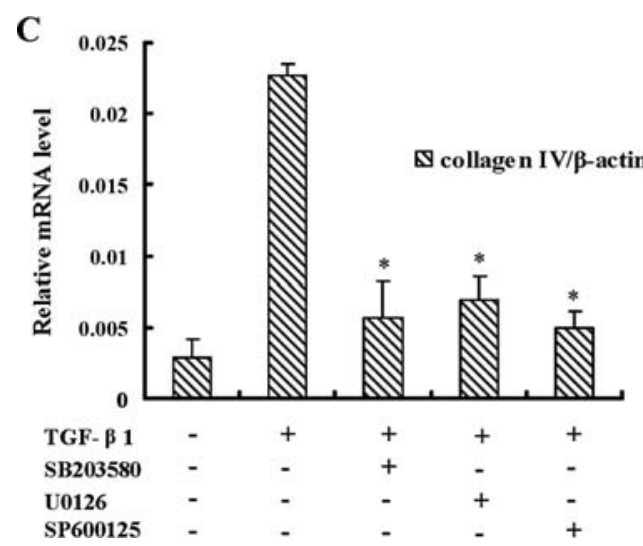

determined by Student's t-test for paired data. Values of $\mathrm{P}<0.05$ were considered significant. Data are presented as means $\pm \mathrm{SD}$ of triplicate experiments.

\section{Results}

TGF- $\beta 1$ induces the activation of ERK1/2, JNK, p38, Smad2 and the expression of collagen IV in rat MCs. Western blot analysis showed that, after treatment with TGF- $\beta 1(2.5 \mathrm{ng} / \mathrm{ml})$, the levels of phosphorylated ERK, JNK, p38 (Fig. 1A), and Smad2 phosphorylated at both linker (Ser245/250/255) and C-terminal (Ser465/467) sites (Fig. 1B) increased rapidly. The level of phosphorylated JNK peaked at $2 \mathrm{~h}$, while the others reached their peak at $1 \mathrm{~h}$ after TGF- $\beta 1$ stimulation. The phosphorylated levels of ERK1/2 and JNK were maintained at a high level for $4 \mathrm{~h}$, and that of p38 and Smad2 for $6 \mathrm{~h}$. Moreover, the expression of collagen IV also increased after treatment with TGF- $\beta 1$ and peaked at $6 \mathrm{~h}$ (Fig. 1C).

Inhibitory effects of three MAPK inhibitors on TGF- $\beta 1$ induced activation of ERK1/2, JNK, $p 38$, and Smad2, and the expression of collagen IV in rat MCs. To explore the effects of MAPK inhibitors on TGF- $\beta 1$ signaling pathways, rat MCs were pretreated with U0126, SP600125 or SB203580, followed by treatment with TGF- $\beta 1(2.5 \mathrm{ng} / \mathrm{ml})$. As shown in
Figure 2. Effects of three MAPK inhibitors on TGF- $\beta 1$-induced activation of ERK1/2, JNK, p38, Smad2 and expression of collagen IV in MCs. Cells were pretreated with the ERK1/2 inhibitor, U0126, JNK inhibitor, SP600125, or p38 inhibitor, SB203580, for $2 \mathrm{~h}$ and then treated with TGF-31 $(2.5 \mathrm{ng} / \mathrm{ml})$ for the indicated times. Protein levels were assessed by Western blotting. (A) Levels of P-ERK1/2, P-JNK, P-p38 MAPK were reduced after treatment with the three respective inhibitors. (B) Effects of three MAPK inhibitors on the phosphorylation of Smad2 at the linker and C-terminal sites, and the expression of collagen IV; $\beta$-actin was used as a loading control. (C) The relative collagen IV mRNA levels, determined by real-time quantitative RTPCR, and expressed as the means \pm SD. ${ }^{*} \mathrm{P}<0.05$, vs. TGF- $\beta 1$. All the results are representative of at least three independent experiments.

Fig. 2A, pretreatment with the three inhibitors all inhibited significantly the phosphorylation of ERK1/2, JNK or p38, respectively. Furthermore, inhibition of ERK by U0126, and inhibition of p38 by SB203580, suppressed the TGF- 31 induced phosphorylation of Smad2 at both the linker and C-terminal sites. Inhibition of JNK by SP600125 only decreased the phosphorylation of Smad2 at the C-terminal sites, but had little effect on the phosphorylation at the linker sites. Moreover, the pretreatment of MCs with the three specific inhibitors did not affect the total amount of Smad2. All three MAPK inhibitors decreased the TGF-ß1-induced expression of collagen IV (Fig. 2B). Real-time quantitative RT-PCR revealed that three MAPK inhibitors, U0126, SP600125 or SB203580, suppressed TGF-ß1-induced collagen IV transcription to $0.25,0.31$ and 0.22 , respectively, of controls obtained with TGF- $\beta 1$ treatment alone $(\mathrm{P}<0.05)$ (Fig. 2C). These findings were consistent with Western blot analysis.

Dominant negative Smad2 inhibits TGF- $\beta 1$-induced phosphorylation of Smad2 and expression of collagen IV. MCs were transiently transfected with a dominant negative Smad 2 construct for $48 \mathrm{~h}$, serum starved for $20 \mathrm{~h}$, and then treated with $2.5 \mathrm{ng} / \mathrm{ml}$ TGF- $\beta 1$. Transient transfection of dominant negative Smad2 inhibited significantly TGF-ß1induced phosphorylation of Smad2 and the expression of 


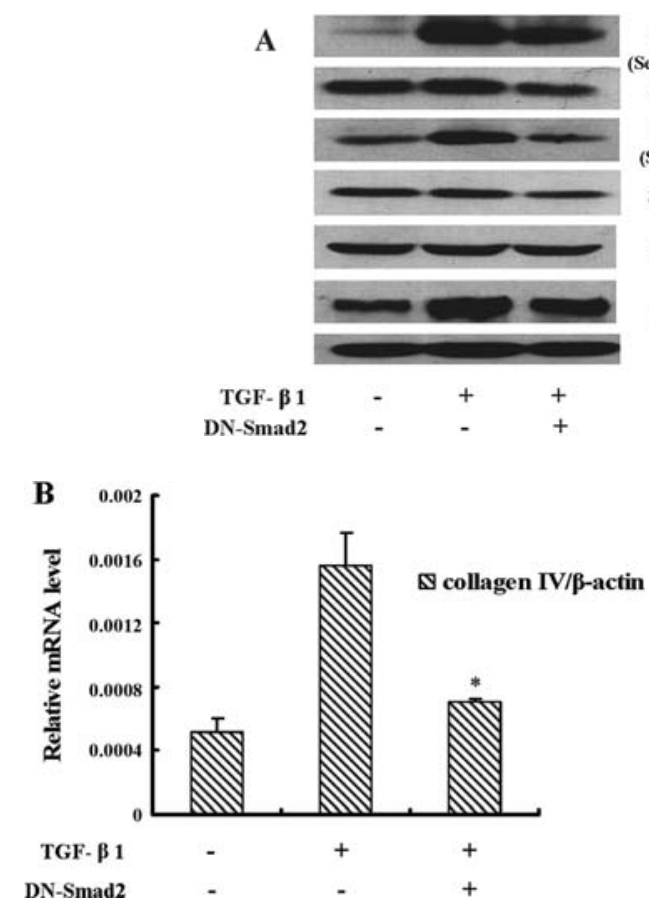

collagen IV (Fig. 3A). Real-time quantitative RT-PCR revealed that dominant negative Smad2 also suppressed TGF- 31 induced transcription of the collagen IV gene to 0.45 of the collagen IV levels after a single TGF- $\beta 1$ treatment. The difference between the two groups was significant $(\mathrm{P}<0.05)$ (Fig. 3B). The real-time quantitative RT-PCR results were consistent with Western blot analysis.

Effects of dominant negative Smad2 on TGF- $\beta 1$-induced phosphorylation of ERK1/2, JNK, p38 MAPK and expression of collagen $I V$. To explore the possibility that blocking Smad2 could also affect MAPK molecules, MCs were transfected transiently with a dominant negative Smad2 construct, as above. As shown in Fig. 4, transient transfection of dominant negative Smad2 inhibited the phosphorylation of ERK1/2 and JNK (Fig. 4A), and the expression of collagen IV (Fig. 4C) induced by TGF- $\beta 1$. However, it did not affect TGF- $\beta 1$ induced phosphorylation of p38 MAPK (Fig. 4B). Moreover, transient transfection of dominant negative Smad2 did not affect the total amount of ERK1/2, JNK and p38. The results suggest that Smad2 may cross-talk with the ERK1/2 and JNK pathways, and thereby influence the synthesis of collagen IV.

\section{Discussion}

We have shown in the present study that blocking the ERK1/2 pathway can reduce the TGF- 11 -induced phosphorylation of Smad 2 at both the linker and C-terminal sites. Recent studies indicate that the interaction between ERK1/2 and Smads, two of the most investigated pathways, is complex, and also controversial in that the interaction can either inhibit $(23,24)$ or enhance $(25,26)$ downstream events. The complex interaction involving several MAPK pathways in regulating Smad activity has also been investigated $(27,28)$. The different responses may be explained by the differences in cellular origin or the method of activation. In human MCs, Hayashida reported a synergic effect between TGF-ß1-activated ERK and Smad3 signaling in collagen production (21). Phosphorylation of the Smad3 linker sites was regulated by the activation of ERK. The structure of Smad2 is similar to that of Smad3, both of which have two conserved domains, MH1 and $\mathrm{MH} 2$, in the $\mathrm{N}$ - and $\mathrm{C}$-terminals, respectively. The MH1 domain is mainly responsible for DNA binding. The $\mathrm{MH} 2$ domain has transcription activity and is also involved in homoand hetero-oligomerization with other Smad proteins. The two domains are joined by a less conserved linker region that is serine/threonine-rich and contains multiple phosphorylation sites for proline-directed kinases $(29,30)$. TGF- 31 stimulates the activation of receptors. The activated receptors then phosphorylate Smad2 and Smad3, forming a Smad2/3 dipolymer. Since activating ERK causes phosphorylation of Smad3, we propose that Smad2 may play a similar role in signal transduction. In the present study, we demonstrated that TGF-ß1induced phosphorylation of Smad2 was decreased by blocking the ERK1/2 pathway using its specific inhibitor. This result suggested that TGF-ß1-dependent ERK1/2 activation enhances the phosphorylation of both Smad2 and Smad3, resulting in a synergic effect on collagen synthesis in MCs.

We further determined that p38 and JNK, the other molecules of in the MAPK pathway, also interact with Smad2. TGF-ß1-induced expression of collagen IV and phosphorylation of Smad 2 at both the linker and C-terminal sites were downregulated by a p38-specific inhibitor. Phosphorylation of Smad2 at the C-terminal sites also decreased, with concomitant reduced expression of collagen IV when a JNK-specific inhibitor was used. These results reveal that, in rat MCs stimulated with TGF-ß1, all three MAPK pathways participate in the interaction between MAPK and Smad2.

The phosphorylation of the SSXS sites of the Smad2/3 Ctermini by the type I receptor of TGF- $\beta$ is critical to enable their association with Smad4 (31,32). Recent reports suggest, however, that other phospho-acceptor sites in Smad2/3 play a 

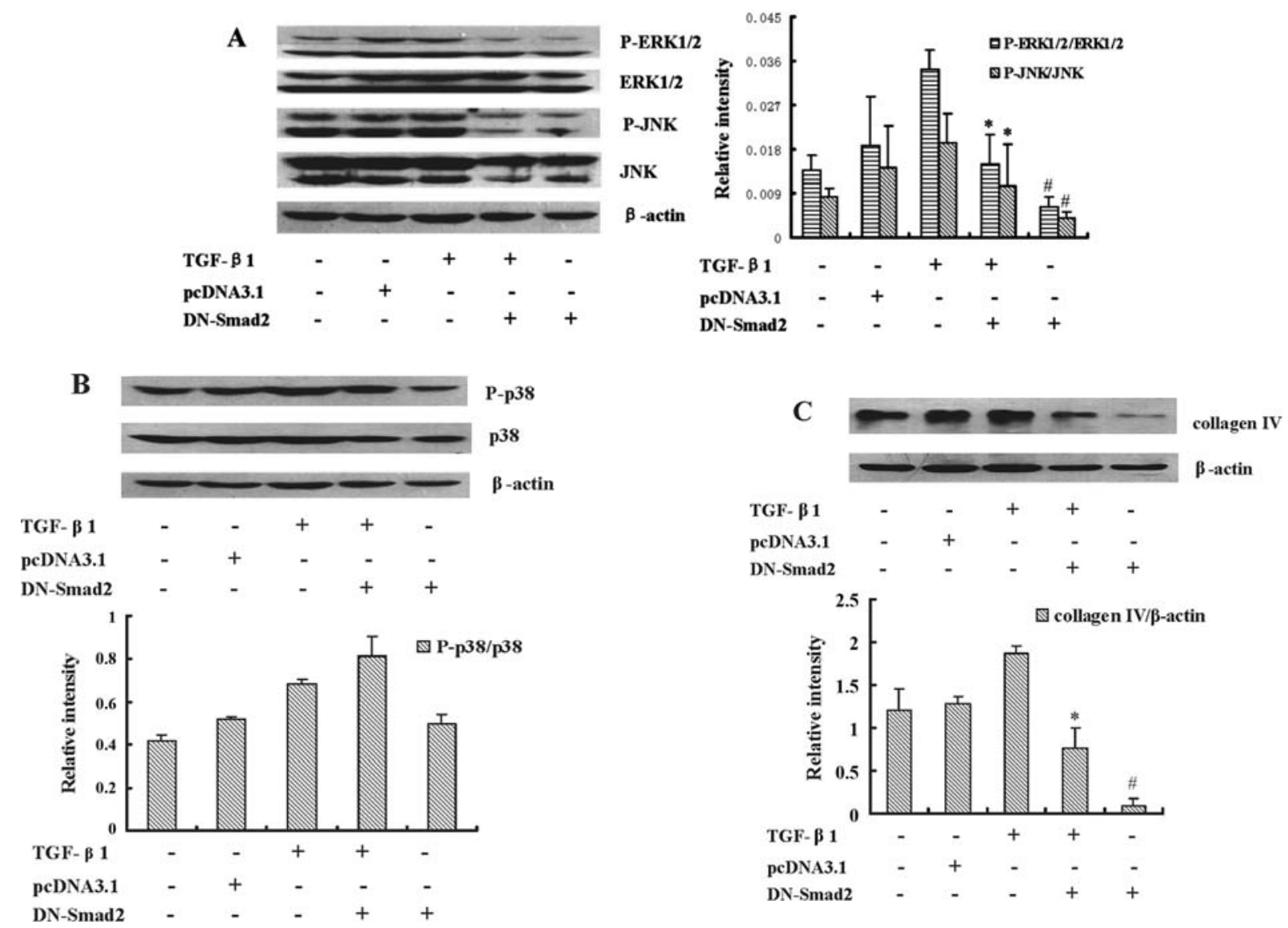

Figure 4. Effects of dominant negative Smad2 on TGF-ß1 induced phosphorylation of ERK1/2, JNK, p38 MAPK and expression of collagen IV. (A, B) Cells were transfected with a dominant negative Smad2 construct using Lipofectamine ${ }^{\mathrm{TM}}$ 2000. Two days after transfection, some cells were stimulated with TGF- 11 for $1 \mathrm{~h}$, and the relative ERK1/2 and P-ERK1/2, JNK and P-JNK, p38 and P-p38, and B-actin protein levels were determined by Western blotting. (C) Remaining cells were stimulated with TGF- $\beta 1$ for $6 \mathrm{~h}$. The relative collagen IV protein levels were determined by Western blotting. B-actin was used as a loading control. Results are representative of at least three replicated experiments. ${ }^{*} \mathrm{P}<0.05$, vs. TGF- $\beta 1$; ${ }^{*} \mathrm{P}<0.05$, vs. normal.

regulatory role in TGF- $\beta$ signaling $(33,34)$. Kretzschmar et al have shown that several ERK consensus phosphorylation sites in the Smad linker region may play a role in the interaction between the two pathways (35). Mutation of these linker sites increased the ability of Smad3 to activate target genes, suggesting that MAPK-induced phosphorylation of the Smad3 linker region has an inhibitory effect (36). By contrast, Hayashida found that the ERK-dependent phosphorylation of the Smad3 linker region enhances collagen I synthesis induced by TGF-B1 (21). Phosphorylation of Smad3 linker sites by p38 MAPK and JNK may enhance Smad2/3 transcriptional activity, implying that Smads and the $\mathrm{p} 38 / \mathrm{JNK}$ signaling pathways collaborate on generating a more robust TGF- $\beta$ response $(16,37)$. The data presented in the above reports indicate that the linker region of $\operatorname{Smad} 2 / 3$ is another important regulatory site mediated by MAPK. Interestingly, we found that the inhibition of ERK and p38 reduced the phosphorylation of Smad2 at both the linker and C-terminal sites, with a synergistic effect on decreasing the synthesis of collagen IV. This observation is consistent with the synergic regulation found in other studies $(11,12,21)$. However, the inhibition of JNK reduced the phosphorylation of Smad2 only at the C-terminal sites, resulting in the reduction of collagen IV synthesis, but it had no obvious effect on the phosphorylation at the linker sites. The complex mechanisms of these differences remain to be elucidated.
So far, the cross-talk between the Smads and MAPK pathways has been investigated widely, and the ability of MAPK to mediate the phosphorylation of Smad has been well established, while the reciprocal ability of Smads to mediate the activation of MAPK has also been demonstrated. A recent report has shown that the ablation of Smad3 can inhibit the activation of JNK and ERK induced by TGF- 31 and v-ras, thereby protecting mouse embryonic fibroblasts from oncogenic transformation (17). We also observed reduced TGF-B1-mediated phosphorylation of JNK and ERK1/2 when dominant negative Smad2 was transfected into rat MCs, leading to a decrease of collagen IV expression. This observation suggested the ability of Smad2 to mediate the MAPK pathway in the reverse direction. Based on our results, we propose that the cross-talk between MAPK and Smad plays an important role in collagen synthesis in rat MCs. Therefore, the present work provides a basis for further investigation in the interaction between $\operatorname{Smad} 2 / 3$ and the other signal transducers, to identify new, useful targets for the prevention and treatment of glomerulonephritis and glomerulosclerosis.

\section{Acknowledgements}

This work was supported by grants from the National Natural Science Foundation of China (NSFC: 30570859), and the 
Shanghai Leading Academic Discipline Project (B110). We thank Dr Chun Peng (Department of Biology, York University, Toronto, Canada) for the kind gift of the dominant negative Smad2 plasmid and Mr Zhonghua Zhao (Department of Pathology, Fudan University) for technical assistance. We also thank International Science Editing for providing language help.

\section{References}

1. Kashgarian M and Sterzel RB: The pathobiology of the mesangium. Kidney Int 41: 524-529, 1992.

2. Leask $A$ and Abraham DJ: TGF- $\beta$ signaling and the fibrotic response. FASEB J 18: 816-827, 2004.

3. Jiang $\mathrm{T}$, Che Q, Lin Y, Li H and Zhang N: Aldose reductase regulates TGF-ß1-induced production of fibronectin and type IV collagen in cultured rat mesangial cells. Nephrology 11: 105-112, 2006.

4. Lambert E, Dassé E, Haye B and Petitfrère E: TIMPs as multifacial proteins. Crit Rev Oncol Hematol 49: 187-198, 2004.

5. Kwak HJ, Park MJ, Cho H, Park CM, Moon SI, Lee HC, Park IC, Kim MS, Rhee $\mathrm{CH}$ and Hong SI: Transforming growth factor- $\beta 1$ induces tissue inhibitor of metalloproteinase-1 expression via activation of extracellular signal-regulated kinase and $\mathrm{Sp} 1$ in human fibrosarcoma cells. Mol Cancer Res 4: 209-220, 2006.

6. Montenegro DE, Franklin T, Moscinski LC, Zuckerman KS and Hu XT: TGF- $B$ inhibits GM-CSF-induced phosphorylation of ERK and MEK in human myeloid leukaemia cell lines via inhibition of phosphatidylinositol 3-kinase (PI3-k). Cell Prolif 42: 1-9, 2009.

7. Runyan CE, Schnaper HW and Poncelet AC: The phosphatidylinositol 3-kinase/Akt pathway enhances Smad3stimulated mesangial cell collagen I expression in response to transforming growth factor- 31 . J Biol Chem 279: 2632-2639, 2004.

8. Javelaud D and Mauviel A: Crosstalk mechanisms between the mitogen-activated protein kinase pathways and Smad signaling downstream of TGF-3: implications for carcinogenesis. Oncogene 24: 5742-5750, 2005.

9. Derynck R and Zhang YE: Smad-dependent and Smadindependent pathways in TGF- $\beta$ family signaling. Nature 425 : 577-584, 2003.

10. Moustakas A and Heldin CH: Non-Smad TGF- $\beta$ signals. J Cell Sci 118: 3573-3584, 2005

11. Li F, Zeng BF, Chai YM, Cai P, Fan C and Cheng T: The linker region of Smad2 mediates TGF- $\beta$-dependent ERK2-induced collagen synthesis. Biochem Biophys Res Commun 386: 289-293, 2009.

12. Li F, Fan CY, Cheng T, Jiang C and Zeng B: Efficient inhibition of fibroblast proliferation and collagen expression by ERK2 siRNAs. Biochem Biophys Res Commun 382: 259-263, 2009.

13. Liu X, Sun SQ, Hassid A and Ostrom RS: cAMP inhibits transforming growth factor- $\beta$-stimulated collagen synthesis via inhibition of extracellular signal-regulated kinase 1/2 and Smad signaling in cardiac fibroblasts. Mol Pharmacol 70: 1992-2003, 2006

14. Sekimoto G, Matsuzaki K, Yoshida K, Mori S, Murata M, Seki T, Matsui H, Fujisawa J and Okazaki K: Reversible Smaddependent signaling between tumor suppression and oncogenesis. Cancer Res 67: 5090-5096, 2007.

15. Matsuzaki K, Kitano C and Murata M: Smad2 and Smad3 phosphorylated at both linker and $\mathrm{COOH}$ terminal regions transmit malignant TGF- $\beta$ signal in later stages of human colorectal cancer. Cancer Res 69: 5321-5330, 2009.

16. Mori S, Matsuzaki K, Yoshida K, Furukawa F, Tahashi Y, Yamagata H, Sekimoto G, Seki T, Matsui H, Nishizawa M, Fujisawa $\mathrm{J}$ and Okazaki K: TGF- $\beta$ and HGF transmit the signals through JNK-dependent Smad2/3 phosphorylation at the linker regions. Oncogene 23: 7416-7429, 2004.

17. Arany PR, Rane SG and Roberts AB: Smad3 deficiency inhibits $\mathrm{v}$-ras-induced transformation by suppression of JNK MAPK signaling and increased farnesyl transferase inhibition. Oncogene 27: 2507-2512, 2008.
18. Ohshima $\mathrm{T}$ and Shimotohno K: Transforming growth factor- $\beta$ mediated signaling via the p38 MAP kinase pathway activates Smad-dependent transcription through SUMO-1 modification of Smad4. J Biol Chem 278: 50833-50842, 2003.

19. Ungefroren H, Lenschow W and Chen WB: Regulation of biglycan gene expression by transforming growth factor- $\beta$ requires MKK6-p38 mitogen-activated protein kinase signaling downstream of Smad signaling. J Biol Chem 278: 11041-11049, 2003.

20. Schnaper HW, Hayashida T, Hubchak SC and Poncelet AC: TGF- $\beta$ signal transduction and mesangial cell fibrogenesis. Am J Physiol Renal Physiol 284: F243-F252, 2003.

21. Hayashida T, deCaestecker M and Schnaper HW: Cross-talk between ERK MAP kinase and Smad-signaling pathways enhances TGF- $\beta$ dependent responses in human mesangial cells. FASEB J 17: 1576-1580, 2003.

22. Wu H, Wang S, Xue A, Liu Y, Liu Y, Wang H, Chen Q, Guo M and Zhang Z: Overexpression of decorin induces apoptosis and cell growth arrest in cultured rat mesangial cells in vitro. Nephrology 13: 607-615, 2008.

23. Kretzschmar M, Doody J, Timokhina I and Massagué J: A mechanism of repression of TGFß/Smad signaling by oncogenic Ras. Genes Dev 13: 804-816, 1999.

24. Calonge MJ and Massagué J: Smad4/DPC4 silencing and hyperactive Ras jointly disrupt transforming growth factor- $\beta$ antiproliferative responses in colon cancer cells. J Biol Chem 274: 33637-33643, 1999.

25. Watanabe H, de Caestecker MP and Yamada Y: Transcriptional cross-talk between Smad, ERK1/2, and p38 mitogen-activated protein kinase pathways regulates transforming growth factor- $\beta$ induced aggrecan gene expression in chondrogenic ATDC5 cells. J Biol Chem 276: 14466-14473, 2001.

26. Beier F, Taylor AC and LuValle P: The Raf-1/MEK/ERK pathway regulates the expression of the $\mathrm{p} 21$ (Cip1/Waf1) gene in chondrocytes. J Biol Chem 274: 30273-30279, 1999.

27. Ravanti L, Häkkinen L, Larjava H, Saarialho-Kere U, Foschi M, Han J and Kähäri VM: Transforming growth factor- $\beta$ induces collagenase-3 expression by human gingival fibroblasts via p38 mitogen-activated protein kinase. J Biol Chem 274: 37292-37300, 1999.

28. Peron P, Rahmani M, Zagar Y, Durand-Schneider AM, Lardeux B and Bernuau D: Potentiation of Smad transactivation by Jun proteins during a combined treatment with epidermal growth factor and transforming growth factor- $\beta$ in rat hepatocytes. Role of phosphatidylinositol 3-kinase-induced AP-1 activation. J Biol Chem 276: 10524-10531, 2001.

29. Massagué J: TGF-ß signal transduction. Annu Rev Biochem 67: 753-791, 1998.

30. Heldin $\mathrm{CH}$, Miyazono $\mathrm{K}$ and ten Dijke P: TGF-beta signalling from cell membrane to nucleus through SMAD proteins. Nature 390: 465-471, 1997.

31. Abdollah S, Macías-Silva M, Tsukazaki T, Hayashi H, Attisano L and Wrana JL: TbetaRI phosphorylation of Smad2 on Ser465 and Ser467 is required for Smad2-Smad4 complex formation and signaling. J Biol Chem 272: 27678-27685, 1997.

32. Souchelnytskyi S, Tamaki K, Engström U, Wernstedt C, Ten Dijke P and Heldin CH: Phosphorylation of Ser465 and Ser467 in the $C$ terminus of Smad2 mediates interaction with Smad4 and is required for transforming growth factor-beta signaling. J Biol Chem 272: 28107-28115, 1997.

33. Yakymovych I, Ten Dijke P, Heldin CH and Souchelnytskyi S: Regulation of Smad signaling by protein kinase C. FASEB J 15: 553-555, 2001

34. Wicks SJ, Lui S, Abdel-Wahab N, Mason RM and Chantry A: Inactivation of smad-transforming growth factor beta signaling by $\mathrm{Ca}(2+)$-calmodulin-dependent protein kinase II. Mol Cell Biol 20: 8103-8111, 2000.

35. Kretzschmar M, Doody J and Massagué J: Opposing BMP and EGF signaling pathways converge on the TGF- $B$ family mediator Smad1. Nature 389: 618-622, 1997.

36. Matsuura I, Wang G, He D and Liu F: Identification and characterization of ERK MAP kinase phosphorylation sites in Smad3. Biochemistry 44: 12546-12553, 2005.

37. Kamaraju AK and Roberts AB: Role of Rho/ROCK and p38 MAP kinase pathways in transforming growth factor-betamediated Smad-dependent growth inhibition of human breast carcinoma cells in vivo. J Biol Chem 280: 1024-1036, 2005. 